\title{
Diferentes Substratos para a Produção de Mudas de Sesbania virgata
}

\author{
William Macedo Delarmelina ${ }^{1}$, Marcos Vinicius Winckler Caldeira ${ }^{1}$, \\ Júlio Cézar Tannure Faria ${ }^{2}$, Elzimar de Oliveira Gonçalves ${ }^{1}$, Rafael Luiz Frinhani Rocha ${ }^{3}$ \\ ${ }^{1}$ Departamento de Ciências Florestais e da Madeira, Centro de Ciências Agrárias, \\ Universidade Federal do Espírito Santo - UFES, Alegre/ES, Brasil \\ ${ }^{2}$ Departamento de Ciências Florestais, Universidade Federal Rural do Rio de Janeiro - UFRRJ, Seropédica/RJ, Brasil \\ ${ }^{3}$ Instituto Federal de Educação, Ciência e Tecnologia do Espírito Santo - IFES, Campus de Alegre, Alegre/ES, Brasil
}

\begin{abstract}
RESUMO
A produção de mudas de espécies florestais nativas visa a atender aos plantios, principalmente para fins ambientais, tais como a recuperação de áreas degradadas. Para intensificar os conhecimentos sobre espécies com potencial de recuperação de áreas degradadas, este trabalho teve como objetivo avaliar a utilização de diferentes substratos no crescimento de mudas de Sesbania virgata (Cav.) Pers. Os tratamentos foram formulados utilizando-se lodo de esgoto, fibra de coco, vermiculita e casca de arroz in natura, além do substrato comercial. As mudas foram produzidas em tubetes com capacidade para $120 \mathrm{~cm}^{3}$. O estudo foi constituído de 14 tratamentos, com cinco repetições de cinco mudas cada, totalizando 25 plantas cada tratamento. Após 150 dias da semeadura, foram avaliadas as seguintes características nas mudas: altura; diâmetro do coleto; relação entre a altura da parte aérea e o diâmetro do coleto; massa seca da parte aérea; massa seca do sistema radicular; massa seca total; relação entre a massa seca da parte aérea e a massa seca do sistema radicular, e índice de qualidade de Dickson. Os tratamentos que continham lodo de esgoto e vermiculita em sua composição, sobretudo o tratamento T6 (60\% lodo de esgoto $+40 \%$ vermiculita), proporcionaram o melhor crescimento das características morfológicas das mudas de Sesbania virgata.
\end{abstract}

Palavras-chave: caraterísticas morfológicas, qualidade de mudas, resíduos.

\section{Different Substrates for the Production of Sesbania virgata Seedlings}

\begin{abstract}
The native seedlings production aims to attend plantings, mainly for environmental purposes, such as the recovery of degraded areas. To enhance the knowledge about species with potential for recovery of degraded areas, this study aimed to evaluate the use of different substrates on the growth of seedlings of Sesbania virgata (Cav.) Pers. The treatments were formulated using sewage sludge, coconut fiber, vermiculite and rice husk in natura, besides the commercial substrate. The seedlings were grown in plastic tubes with a capacity of $120 \mathrm{~cm}^{3}$. The study consisted of fourteen treatments, with five replicates of five seedlings each, in a total of 25 plants per treatment. After 150 days of sowing, the following characteristics were evaluated in seedling: height, stem base diameter, the relation between height and the stem base diameter, shoot dry matter, root dry matter, total dry matter, the relation between the shoot dry matter and root dry matter and Dickson quality index. The treatments containing sewage sludge and vermiculite in its composition, particularly the treatment T6 (60\% sewage sludge $+40 \%$ vermiculite), provided the best growth of morphological characteristics of seedlings of Sesbania virgata.
\end{abstract}

Keywords: morphological characteristics, seedling quality, waste. 


\section{INTRODUÇÃO}

Mudas de espécies florestais nativas são produzidas em viveiro, com o objetivo de serem usadas em recuperação de áreas degradadas, reflorestamento e soluções ambientais. Neste contexto, a produção de mudas com o intuito de recuperar áreas impactadas possui extrema importância, em razão da intensa devastação das florestas nativas, que é devida, principalmente, à expansão urbana e das fronteiras agropecuárias.

A produção de mudas de qualidade depende de vários fatores, sendo a composição dos substratos um fator de grande importância, pois a germinação de sementes, a iniciação radicular e o enraizamento estão diretamente ligados às características químicas, físicas e biológicas do substrato (Caldeira et al., 2000). Nos viveiros florestais, é comum a utilização de componentes orgânicos para a produção de mudas com o objetivo de melhorar os atributos físicos, químicos e biológicos dos substratos; porém, de forma geral, as formulações dos substratos são pobres em nutrientes essenciais para o crescimento da planta, sendo a adubação mineral necessária para que as mudas se desenvolvam de maneira satisfatória.

Devido a isso, o estudo detalhado da melhor combinação de materiais para compor um substrato a fim de promover maior crescimento inicial de mudas com qualidade e de forma rápida é fundamental para melhorar a produção na fase de viveiro (Morais et al., 1996). Diversos materiais podem ser utilizados na composição dos substratos para a produção de mudas, como, por exemplo, o composto com casca de pinus semidecomposta, que atualmente é utilizado em larga escala para a produção de mudas florestais e em diversas áreas agrícolas. Contudo, alguns estudos sugerem que a sua utilização para produção de diversas espécies florestais não seria indicada (Caldeira et al., 2012; Peroni, 2012).

Neste contexto, insere-se, como alternativa para a produção de mudas de qualidade e de baixo custo, o lodo de esgoto, pois este pode ser usado como condicionador dos atributos físicos, químicos e biológicos do solo, considerando seu teor de matéria orgânica e nutrientes (Melo et al., 1994), além de promover o benefício ambiental pela reutilização deste resíduo.
Para recuperação de áreas degradadas, de uma maneira geral, recomendam-se espécies de rápido crescimento, rústicas e fixadoras de nitrogênio. Com isso, a espécie Sesbania virgata possui características viáveis para ser utilizada em áreas impactadas, por pertencer à família Leguminosae, sendo considerada uma espécie pioneira, arbustiva e semiperene, que forma simbiose radicular com Azorhizobium spp. (Santos et al., 1997). Em estudo realizado por Chaves et al. (2003), os autores mencionam que esta espécie apresenta alto potencial para utilização em programas de recuperação de áreas degradadas, haja vista a facilidade de propagação dessa espécie, no que se refere às altas taxas de crescimento e cobertura que é capaz de promover no solo. Portanto, este trabalho teve como objetivo avaliar diferentes proporções de lodo de esgoto, fibra de coco, vermiculita e casca de arroz in natura como substrato na produção de mudas de Sesbania virgata.

\section{MATERIAL E MÉTODOS}

As mudas de Sesbania virgata foram produzidas no Viveiro Florestal do Centro de Ciências Agrárias da Universidade Federal do Espírito Santo (UFES), durante o período de maio a setembro de 2011, estando localizado na Área Experimental I no município de Alegre-ES. O clima da região enquadrase no tipo Cwa (inverno seco e verão chuvoso), de acordo com a classificação de Köppen, sendo a temperatura média anual de $24,1^{\circ} \mathrm{C}$, com máximas diárias de $31^{\circ} \mathrm{C}$ e mínimas de $20,2^{\circ} \mathrm{C}$, e precipitação anual média de $1.104 \mathrm{~mm}$ (Maia et al., 2007).

Os tratamentos foram formulados utilizando-se lodo de esgoto (LE), fibra de coco (FC), vermiculita (VERM) e casca de arroz in natura (CA in natura), além do substrato comercial (SC) composto de $60 \%$ de composto de casca de pinus, $15 \%$ de vermiculita e $25 \%$ de húmus mais terra vegetal, utilizado como tratamento testemunha. O lodo de esgoto utilizado na formulação dos substratos é proveniente de filtro anaeróbio de fluxo ascendente e foi doado pela empresa Foz do Brasil S.A., sendo oriundo da Estação de Tratamento de Esgoto da empresa localizada no município de Cachoeiro de Itapemirim-ES. O resultado da análise química (Tabela 1) demonstrou que o lodo de esgoto possui aptidão para uso em ambientes agrícolas, exceto 
Tabela 1. Análise química do lodo de esgoto de filtro anaeróbico oriundo da Estação de Tratamento de Esgoto de Cachoeiro de Itapemirim, ES.

Table 1. Chemical analysis of sewage sludge from the anaerobic filter originating from the sewage treatment plant in Cachoeiro de Itapemirim, ES.

\begin{tabular}{|c|c|c|}
\hline Parâmetros & Resultados Analíticos $^{1}$ & Resolução nº 375/2006 CONAMA ${ }^{2}$ \\
\hline Arsênio & $<0,5 \mathrm{mg} \mathrm{dm}^{-3}$ & $41 \mathrm{mg} \mathrm{kg}^{-1}$ \\
\hline Bário & $156 \mathrm{mg} \mathrm{dm}^{-3}$ & $1300 \mathrm{mg} \mathrm{kg}^{-1}$ \\
\hline Cádmio & $<0,053 \mathrm{mg} \mathrm{dm}^{-3}$ & $39 \mathrm{mg} \mathrm{kg}^{-1}$ \\
\hline Chumbo & $29 \mathrm{mg} \mathrm{dm}^{-3}$ & $300 \mathrm{mg} \mathrm{kg}^{-1}$ \\
\hline Cobre & $98 \mathrm{mg} \mathrm{dm}^{-3}$ & $1500 \mathrm{mg} \mathrm{kg}^{-1}$ \\
\hline Cromo & $26 \mathrm{mg} \mathrm{dm}^{-3}$ & $1000 \mathrm{mg} \mathrm{kg}^{-1}$ \\
\hline Molibdênio & $3,5 \mathrm{mg} \mathrm{dm}^{-3}$ & $50 \mathrm{mg} \mathrm{kg}^{-1}$ \\
\hline Níquel & $11 \mathrm{mg} \mathrm{dm}^{-3}$ & $420 \mathrm{mg} \mathrm{kg}^{-1}$ \\
\hline Selênio & $<0,5 \mathrm{mg} \mathrm{dm}^{-3}$ & $100 \mathrm{mg} \mathrm{kg}^{-1}$ \\
\hline Zinco & $409 \mathrm{mg} \mathrm{dm}^{-3}$ & $2800 \mathrm{mg} \mathrm{kg}^{-1}$ \\
\hline Fósforo Total & $4128 \mathrm{mg} \mathrm{dm}^{-3}$ & - \\
\hline pH (Suspensão a 5\%) & 5,2 & - \\
\hline Enxofre & $1,30 \%$ & - \\
\hline Nitrogênio Total Kjeldahl & $5646 \mathrm{mg} \mathrm{dm}^{-3}$ & - \\
\hline Nitrogênio Amoniacal & $60 \mathrm{mg} \mathrm{dm}^{-3}$ & - \\
\hline Carbono Orgânico Total & $16 \%$ & - \\
\hline Potássio & $1623 \mathrm{mg} \mathrm{dm}^{-3}$ & - \\
\hline Sódio & $399 \mathrm{mg} \mathrm{dm}^{-3}$ & - \\
\hline
\end{tabular}

${ }^{1}$ Resultados fornecidos pela Foz do Brasil S.A.; ${ }^{2}$ Limites máximos de concentração exigidos pelo CONAMA 375/2006 (Brasil, 2006).

para culturas alimentícias, segundo resolução CONAMA - 375/2006 (Brasil, 2006).

A fibra de coco, a vermiculita e o substrato comercial foram doados pela Fibria S.A. (Unidade Aracruz). A casca de arroz in natura foi doada por produtores agrícolas do município de Muniz FreireES.

O experimento foi instalado em um delineamento inteiramente casualizado, constituído de 14 tratamentos, com cinco repetições de cinco mudas cada, totalizando 25 mudas para cada tratamento. As composições de todos os tratamentos podem ser conferidas na Tabela 2.

Antes da semeadura, foi realizada a análise química para a determinação dos teores disponíveis de nutrientes nos substratos, conforme método descrito pela Embrapa (2009) (Tabela 3). As análises foram realizadas no Laboratório de Recursos Hídricos/DCFM/CCA-UFES, em Jerônimo Monteiro-ES.

A caracterização física dos substratos formulados com resíduos foi realizada no Laboratório de Substratos do Departamento de Horticultura e
Tabela 2. Substratos formulados para produção de Sesbania virgata (v:v).

Table 2. Substrates formulated for the production of Sesbania virgata (v: v).

\begin{tabular}{|ccccc|}
\hline Tratamento & LE $^{1}$ & FC $^{2}$ & VERM $^{3}$ & $\begin{array}{c}\text { CA } \\
\text { in natura }\end{array}$ \\
\hline T1 & 80 & 20 & - & - \\
T2 & 60 & 40 & - & - \\
T3 & 40 & 60 & - & - \\
T4 & 20 & 80 & - & - \\
T5 & 80 & - & 20 & - \\
T6 & 60 & - & 40 & - \\
T7 & 40 & - & 60 & - \\
T8 & 20 & - & 80 & - \\
T9 & 80 & - & - & 20 \\
T10 & 60 & - & - & 40 \\
T11 & 40 & - & - & 60 \\
T12 & 20 & - & - & 80 \\
T13 & 100 & - & - & - \\
T14 & & Testemunha (SC) ${ }^{(5)}$ & \\
\hline
\end{tabular}

${ }^{1}$ Lodo de esgoto; ${ }^{2}$ fibra de coco; ${ }^{3}$ vermiculita; ${ }^{4}$ casca de arroz in natura $;{ }^{5}$ substrato comercial.

Silvicultura da Universidade Federal do Rio Grande do Sul (UFRGS), conforme metodologia descrita na Instrução Normativa $n^{\circ} 17$ do Ministério da 
Tabela 3. Caracterização química dos substratos formulados com diferentes resíduos na produção de mudas de Sesbania virgata.

Table 3. Chemical characterization of substrates formulated with different wastes in Sesbania virgata seedlings production.

\begin{tabular}{|c|c|c|c|c|c|c|c|c|c|c|c|c|c|c|}
\hline \multirow{2}{*}{ Tratamento } & pH & $\mathbf{P}$ & $\mathbf{K}$ & $\mathbf{N a}$ & $\mathrm{Ca}$ & Mg & Al & $\mathbf{H}+\mathbf{A l}$ & CTC & SB & $\mathbf{V}$ & $\mathbf{m}$ & $\mathrm{CE}$ & TTSS \\
\hline & $\mathrm{H}_{2} \mathrm{O}$ & \multicolumn{3}{|c|}{$\mathrm{mg} \mathrm{dm}^{-3}$} & \multicolumn{6}{|c|}{$\mathrm{cmol} \mathrm{dm}^{-3}$} & \multicolumn{2}{|c|}{$\%$} & $\mathrm{mS} \mathrm{cm}^{-1}$ & $g L^{-1}$ \\
\hline $\mathrm{T} 1$ & 4,0 & 176 & 247 & 63 & 12,3 & 4,6 & 1 & 18,5 & 36,23 & 17,74 & 49 & 5 & 0,89 & 2,76 \\
\hline $\mathrm{T} 2$ & 4,1 & 167 & 526 & 56 & 10,1 & 3,8 & 0,6 & 21,0 & 36,43 & 15,43 & 42 & 4 & 0,75 & 1,85 \\
\hline $\mathrm{T} 3$ & 4,4 & 137 & 699 & 40 & 6,1 & 2,9 & 0,4 & 24,2 & 35,09 & 10,93 & 31 & 4 & 0,66 & 1,20 \\
\hline $\mathrm{T} 4$ & 4,7 & 145 & 1105 & 44 & 4,7 & 2 & 0,3 & 21,7 & 31,3 & 9,65 & 31 & 3 & 0,49 & 0,61 \\
\hline T5 & 4,0 & 154 & 132 & 87 & 17,7 & 5,8 & 0,9 & 19,3 & 43,56 & 24,24 & 56 & 4 & 0,90 & 3,32 \\
\hline T6 & 4,1 & 132 & 125 & 133 & 12,5 & 4,7 & 0,9 & 19,7 & 37,83 & 18,17 & 48 & 5 & 0,60 & 2,01 \\
\hline T7 & 4,0 & 123 & 92 & 151 & 10 & 4,6 & 0,7 & 15,2 & 30,63 & 15,47 & 51 & 4 & 0,54 & 1,47 \\
\hline T8 & 4,4 & 62 & 72 & 191 & 5,7 & 4,3 & 0,7 & 11,3 & 22,37 & 11,05 & 49 & 6 & 0,26 & 0,57 \\
\hline T9 & 4,3 & 182 & 470 & 25 & 13,1 & 3,2 & 0,5 & 23,5 & 41,03 & 17,54 & 43 & 3 & 1,34 & 5,26 \\
\hline T10 & 4,6 & 200 & 834 & 25 & 10,2 & 2,8 & 0,5 & 18,5 & 33,72 & 15,23 & 45 & 3 & 1,14 & 3,60 \\
\hline T11 & 4,9 & 180 & 896 & 20 & 7,5 & 2,1 & 0,3 & 15,5 & 27,47 & 11,97 & 44 & 2 & 0,94 & 2,34 \\
\hline T12 & 5,7 & 204 & 1170 & 20 & 3,9 & 1,9 & 0,4 & 10,2 & 19,01 & 8,85 & 47 & 4 & 0,55 & 0,93 \\
\hline T13 & 4,0 & 204 & 144 & 71 & 12,5 & 4,8 & 0,9 & 23,0 & 41,03 & 18,04 & 44 & 5 & 1,42 & 6,22 \\
\hline T14 & 6,6 & 189 & 470 & 20 & 13,1 & 13,9 & 0,0 & 7,0 & 35,27 & 28,28 & 80 & 0 & 0,94 & 3,17 \\
\hline
\end{tabular}

CTC: capacidade de troca catiônica; SB: soma de bases; V: saturação por bases; m: saturação por alumínio; CE: condutividade elétrica; TTSS: teor total de sais solúveis.

Agricultura, Pecuária e Abastecimento (Brasil, 2007). Foram determinados: densidade aparente (DENS); macroporosidade (MACROP); microporosidade (MICROP); volume total de poros (VTP); espaço de aeração (EA); água facilmente disponível (AFC); água tamponante (AT), e água disponível (AD). Os resultados encontram-se descritos na Tabela 4.

Os dados obtidos no presente trabalho foram comparados com a classificação proposta por Gonçalves \& Poggiani (1996), que estabelece uma escala de valores para a interpretação das características físicas e químicas dos substratos (Tabela 5).

As sementes de Sesbania virgata foram doadas pela Reserva Natural da Vale e passaram por um processo de quebra de dormência em água, à temperatura ambiente, por embebição durante 15 minutos. Posteriormente, realizou-se a semeadura de três sementes diretamente no tubete.

As mudas foram produzidas em tubetes com capacidade para $120 \mathrm{~cm}^{3}$ de substrato e acondicionadas em bandejas de polipropileno com capacidade de 54 tubetes, sendo estas bandejas dispostas em canteiros suspensos a $80 \mathrm{~cm}$ do solo, em casa de sombra. Após a germinação $( \pm 20$ dias após semeadura), foi realizado o desbaste, deixando uma muda por recipiente. A irrigação foi realizada por microaspersores quatro vezes ao dia, por sistema de irrigação automático, sendo realizadas duas irrigações na parte da manhã e duas na parte da tarde.

Aos 150 dias após a semeadura, o experimento foi encerrado e foram mensuradas as seguintes características: altura $(\mathrm{H})$, diâmetro do coleto (DC), relação altura/diâmetro coleto (H/DC), massa seca da parte aérea (MSPA), massa seca radicular (MSR), massa seca total (MST), relação massa seca da parte aérea/massa seca radicular (MSPA/MSR) e Índice de Qualidade de Dickson (IQD). O diâmetro do coleto foi obtido com paquímetro digital e a altura, com régua milimetrada, tomando-se como padrão a gema terminal (meristema apical). A quantificação da massa seca da parte aérea e da massa seca do sistema radicular foi realizada por meio da pesagem das partes vegetais, após a secagem em estufa de circulação de ar forçada a $70^{\circ} \mathrm{C}$, por um período de aproximadamente $72 \mathrm{~h}$. O Índice de Qualidade de Dickson foi obtido pela Equação (1) de Dickson et al. (1960):

$I Q D=\frac{\mathrm{MST}_{(\mathrm{g})}}{\mathrm{H}(\mathrm{cm}) / \mathrm{DC}(\mathrm{mm})+\operatorname{MSPA}(\mathrm{g}) / \mathrm{MSR}_{(\mathrm{g})}}$ 
Em que:

- $\operatorname{MST}(\mathrm{g})$ = Massa seca total;

- $\mathrm{H}(\mathrm{cm})=$ Altura;

- $\mathrm{DC}(\mathrm{mm})=$ Diâmetro do coleto;

- $\operatorname{MSPA}(\mathrm{g})$ = Massa seca da parte aérea;

- $\operatorname{MSR}(\mathrm{g})=$ Massa seca da raiz.

Os dados das características mensuradas das 25 mudas de cada tratamento foram submetidos à análise estatística por agrupamento de médias pelo teste Scott-Knott no nível de 5\% de significância, para averiguar diferença estatística entre as médias.

\section{RESULTADOS E DISCUSSÃO}

Verificou-se que os substratos contendo menores proporções de lodo de esgoto influenciaram negativamente no crescimento das mudas para a característica altura (Tabela 6). A maior média para

Tabela 4. Caracterização física dos substratos formulados com resíduos na produção de mudas de Sesbania virgata. Table 4. Physical characterization of substrates formulated with waste in the production of seedlings of Sesbania virgate.

\begin{tabular}{|cccccccccc|}
\hline \multirow{2}{*}{ Tratamento } & DENS & MACROP & MICROP & VTP & EA & AFD & AT & AD \\
\cline { 2 - 9 } & $\mathbf{g ~ c m}^{-3}$ & & & \% & & & & \\
T1 & 0,23 & 22 & 54 & 76 & 17 & 26 & 6 & 31 \\
T2 & 0,17 & 20 & 52 & 72 & 12 & 28 & 6 & 34 \\
T3 & 0,11 & 19 & 46 & 65 & 9 & 25 & 6 & 31 \\
T4 & 0,07 & 17 & 30 & 47 & 9 & 15 & 3 & 18 \\
T5 & 0,26 & 27 & 52 & 79 & 21 & 20 & 5 & 26 \\
T6 & 0,19 & 32 & 49 & 81 & 22 & 17 & 6 & 22 \\
T7 & 0,11 & 38 & 44 & 82 & 21 & 12 & 6 & 17 \\
T8 & 0,05 & 40 & 41 & 81 & 19 & 11 & 5 & 16 \\
T9 & 0,18 & 20 & 53 & 73 & 25 & 20 & 4 & 24 \\
T10 & 0,13 & 17 & 48 & 65 & 28 & 14 & 3 & 17 \\
T11 & 0,11 & 16 & 53 & 69 & 41 & 9 & 1 & 10 \\
T12 & 0,05 & 15 & 48 & 63 & 43 & 5 & 0 & 5 \\
\hline T13 & 0,21 & 23 & 52 & 75 & 17 & 25 & 5 & 30 \\
\hline T14 & 0,32 & 33 & 52 & 85 & 26 & 19 & 3 & 23 \\
\hline
\end{tabular}

Densidade aparente (DENS); porosidade total (PT); espaço de aeração (EA); macroporosidade (MACROP); microporosidade (MICROP); água facilmente disponível (AFD); água tamponante (AT); água disponível (AD).

Tabela 5. Escala de valores para interpretação de propriedades físicas e químicas de substratos usados para produção de mudas florestais.

Table 5. Scale for interpretation of physical and chemical properties of substrates used for seedlings production.

\begin{tabular}{|c|c|c|c|c|}
\hline \multirow{2}{*}{ Propriedades } & \multicolumn{4}{|c|}{ Nível } \\
\hline & Baixo & Médio & Alto & Adequado \\
\hline \multicolumn{5}{|l|}{ Físicas } \\
\hline Densidade global $\left(\mathrm{g} \mathrm{cm}^{-3}\right)$ & $<0,25$ & $0,25-0,50$ & $>0,50$ & $0,45-0,55$ \\
\hline Porosidade total (\%) & $<55$ & $55-75$ & $>75$ & $75-85$ \\
\hline macroporosidade (\%) & $<20$ & $20-40$ & $>40$ & $35-45$ \\
\hline microporosidade (\%) & $<25$ & $25-50$ & $>50$ & $45-55$ \\
\hline Capacidade máxima de retenção de água $\left(\mathrm{mL} 50 \mathrm{~cm}^{-3}\right)$ & $<15$ & $15-25$ & $>25$ & $20-30$ \\
\hline \multicolumn{5}{|l|}{ Químicas } \\
\hline Relação $\mathrm{C}$ total/N total & 8 a $12 / 1$ & 12 a $18 / 1$ & $>18 / 1$ & 8 a $12 / 1$ \\
\hline $\mathrm{pH} \mathrm{em} \mathrm{CaCl}_{2} 0,01 \mathrm{M}$ & $<5,0$ & $5,0-6,0$ & $>6,0$ & $5,5-6,5$ \\
\hline $\mathrm{P}$ resina $\left(\mathrm{mg} \mathrm{dm}^{-3}\right)$ & $<200$ & $200-400$ & $>400$ & $400-800$ \\
\hline $\mathrm{K}$ trocável $\left(\mathrm{mmol}_{\mathrm{c}} \mathrm{dm}^{-3}\right)$ & $<587$ & $587-1174$ & $>1174$ & $1174-3911$ \\
\hline Ca trocável $\left(\mathrm{mmol}_{c} \mathrm{dm}^{-3}\right)$ & $<100$ & $100-150$ & $>150$ & $100-200$ \\
\hline $\mathrm{Mg}$ total $\left(\mathrm{mmol}_{\mathrm{c}} \mathrm{dm}^{-3}\right)$ & $<50$ & $50-100$ & $>100$ & $50-100$ \\
\hline CTC efetiva $\left(\mathrm{mmol}_{\mathrm{c}} \mathrm{dm}^{-3}\right)$ & $<100$ & $100-200$ & $>200$ & $>200$ \\
\hline
\end{tabular}

Fonte: adaptado de Gonçalves \& Poggiani (1996). 
altura $(23,57 \mathrm{~cm})$ foi observada com a formulação de $60 \%$ de lodo de esgoto com $40 \%$ de vermiculita, sendo verificado que maiores proporções de lodo de esgoto favorecem o crescimento em altura da mudas de Sesbania virgata.

Observa-se que o volume total de poros (Tabela 4) é considerado adequado nos tratamentos que obtiveram melhores resultados para altura (T1, T5, T6 e T13) e, quando se analisa a relação entre as características químicas e o crescimento da parte aérea, verifica-se que valores adequados de cálcio, segundo Gonçalves \& Poggiani (1996), proporcionam maior crescimento em altura. Em trabalho realizado por Coutinho et al. (2005), ao avaliarem o crescimento inicial de mudas de S. virgata plantadas em uma área degradada pela extração de argila, comprovaram o efeito benéfico da adição de lodo de esgoto, com os seguintes teores: $\mathrm{Ca}^{2+}-9,7 \mathrm{cmol}_{\mathrm{c}} \mathrm{dm}^{-3} ; \mathrm{Mg}^{2+}-2,3 \mathrm{cmol}_{\mathrm{c}} \mathrm{dm}^{-3}$;

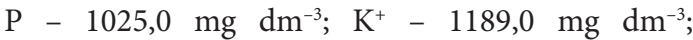
$\mathrm{Cu}$ - 4,2 mg dm${ }^{-3} ; \mathrm{Mn}$ - 48,3 mg $\mathrm{dm}^{-3}$; $\mathrm{Zn}-33,0 \mathrm{mg} \mathrm{dm}{ }^{-3} ; \mathrm{Fe}-78,0 \mathrm{mg} \mathrm{dm}^{-3}$, e conteúdo de matéria orgânica de $47,6 \mathrm{~g} \mathrm{dm}^{-3}$, nas covas de plantio.

Corroborando com os resultados obtidos no presente estudo, Peroni (2012), testando vários componentes - fibra de coco, composto orgânico, casca de arroz in natura e palha de café in natura - associados ao lodo de esgoto, observou crescimento em altura inferior nos tratamentos utilizando fibra de coco e casca de arroz in natura; observou também que as maiores médias foram obtidas com a utilização do composto orgânico juntamente com o lodo de esgoto, para produção de mudas de Eucalyptus grandis. Assim como no presente estudo, Caldeira et al. (2012) verificaram que altas proporções de lodo de esgoto no substrato influencia positivamente a produção de mudas de timbó (Ateleia glazioveana Baill).

De acordo com Daniel et al. (1997) e Carneiro (1995), o diâmetro do coleto é o mais indicado para avaliar a capacidade de sobrevivência da muda no campo, além de ser, também, o mais usado para auxiliar na determinação das doses de fertilizantes a serem aplicadas na produção de mudas. Com base nisso, mudas produzidas nos tratamentos T1, T5 e T6 possuem maior probabilidade de sobrevivência em campo, pois estas apresentaram maiores valores de diâmetro. Diversamente, mudas de S. virgata conduzidas em substratos com alta proporção (>60\%) de fibra de coco, vermiculita e casca de arroz

Tabela 6. Altura (H), diâmetro do coleto (DC), massa seca da parte aérea (MSPA), massa seca de raízes (MSR) e massa seca total (MST) de mudas de Sesbania virgata, 150 dias após a semeadura.

Table 6. Height (H), stem diameter (DC), shoot dry mass (MSPA), root dry mass (MSR) and total dry mass (MST) of Sesbania virgata seedlings, 150 days after sowing.

\begin{tabular}{|c|c|c|c|c|c|}
\hline Tratamento & $\mathbf{H}(\mathbf{c m})$ & $\mathrm{DC}(\mathbf{m m})$ & $\begin{array}{c}\text { MSPA } \\
\left(\text { g planta }^{-1}\right)\end{array}$ & $\begin{array}{c}\text { MSR } \\
\left(\text { g planta }^{-1}\right)\end{array}$ & $\begin{array}{c}\text { MST } \\
\left(\text { g planta }^{-1}\right)\end{array}$ \\
\hline $\mathrm{T} 1$ (80\% LE+20\% FC) & $18,99^{\mathrm{c} 1}$ & $4,26^{\mathrm{a}}$ & $1,528^{c}$ & $0,778^{\mathrm{b}}$ & $2,306^{b}$ \\
\hline $\mathrm{T} 2(60 \% \mathrm{LE}+40 \% \mathrm{FC})$ & $16,61^{\mathrm{d}}$ & $3,30^{d}$ & $0,882^{d}$ & $0,427^{c}$ & $1,299^{c}$ \\
\hline T3 (40\% LE+60\% FC) & $14,72^{\mathrm{e}}$ & $3,65^{c}$ & $0,881^{\mathrm{d}}$ & $0,463^{c}$ & $1,344^{\mathrm{c}}$ \\
\hline T4 (20\% LE+80\% FC) & $10,62^{g}$ & $3,12^{\mathrm{e}}$ & $0,370^{e}$ & $0,231^{\mathrm{c}}$ & $0,601^{d}$ \\
\hline T5 (80\% LE+20\% VERM) & $20,07^{b}$ & $4,43^{\text {a }}$ & $1,708^{\mathrm{b}}$ & $1,184^{\mathrm{a}}$ & $2,892^{\text {a }}$ \\
\hline T6 (60\% LE+40\% VERM) & $23,57^{a}$ & $4,59^{\mathrm{a}}$ & $2,110^{a}$ & $1,203^{\text {a }}$ & $3,313^{\text {a }}$ \\
\hline T7 (40\% LE+60\% VERM) & $18,27^{\mathrm{c}}$ & $3,96^{\mathrm{b}}$ & $1,347^{c}$ & $1,007^{\mathrm{a}}$ & $2,354^{b}$ \\
\hline T8 (20\% LE+80\% VERM) & $15,21^{\mathrm{e}}$ & $3,37^{d}$ & $0,795^{d}$ & $0,631^{b}$ & $1,426^{c}$ \\
\hline T9 (80\% LE+20\% CA in natura) & $16,34^{\mathrm{d}}$ & $4,06^{\mathrm{b}}$ & $1,327^{c}$ & $1,032^{a}$ & $2,359^{b}$ \\
\hline T10 (60\% LE+40\% CA in natura) & $12,23^{\mathrm{f}}$ & $3,05^{\mathrm{e}}$ & $0,883^{d}$ & $0,709^{\mathrm{b}}$ & $1,591^{\mathrm{c}}$ \\
\hline T11 (40\% LE+60\% CA in natura) & $12,83^{\mathrm{f}}$ & $2,90^{\mathrm{e}}$ & $0,623^{d}$ & $0,642^{\mathrm{b}}$ & $1,269^{\mathrm{c}}$ \\
\hline T12 (20\% LE+80\% CA in natura) & $7,30^{\mathrm{h}}$ & $2,41^{\mathrm{f}}$ & $0,266^{e}$ & $0,338^{c}$ & $0,604^{\mathrm{d}}$ \\
\hline T13 (100\% LE) & $18,86^{\mathrm{c}}$ & $4,06^{\mathrm{b}}$ & $1,197^{c}$ & $0,911^{\mathrm{a}}$ & $2,108^{b}$ \\
\hline T14 (100\% SC) & $12,96^{\mathrm{f}}$ & $3,34^{\mathrm{d}}$ & $0,795^{d}$ & $0,543^{b}$ & $1,337^{c}$ \\
\hline $\mathrm{F}$ & ** & ** & ** & ** & ** \\
\hline $\mathrm{CV} \%$ & 4,95 & 6,08 & 13,02 & 24,16 & 14,07 \\
\hline
\end{tabular}

${ }^{1}$ Médias seguidas de letras iguais, na coluna, não diferem entre si pelo teste de Scott - Knott, em $5 \%$ de significância. ${ }^{* * \text { Significativo }}$ $(\mathrm{P}<0,05)$. 
in natura, e no tratamento testemunha não possuem boa estimativa de sobrevivência em campo.

Verificou-se que, embora Valeri \& Corradini (2005) tenham sugerido que o potássio, além de regular a abertura estomática, promove $o$ engrossamento do caule das mudas, no presente estudo, esta associação não ficou evidente, já que as maiores médias de diâmetro de coleto foram verificadas nos tratamento cujos teores de potássio (Tabela 3) são considerados baixos, de acordo com a classificação de Gonçalves \& Poggiani (1996). Em estudo feito por Gonçalves et al. (2000), considerase o valor situado entre 5 e $10 \mathrm{~mm}$ de diâmetro do coleto adequado a mudas de espécies florestais com bom padrão de qualidade. No presente estudo, nenhum tratamento proporcionou o incremento dentro dos limites avaliados ideais pelos autores supracitados, sendo encontrada uma variação entre 2,41 e 4,59 mm; isso indica que os valores propostos não sejam considerados aplicáveis para mudas de $S$. virgata ou que as mudas necessitam de uma maior permanência no viveiro para então irem ao campo.

A massa seca da parte aérea, segundo Gomes \& Paiva (2006), indica a rusticidade de uma muda, sendo que os maiores valores representam mudas mais lignificadas e rústicas, tendo maior aproveitamento em ambientes com condições adversas. Para MSPA, os valores variaram de $0,27 \mathrm{~g}$ para o tratamento T12 a 2,11 g para o tratamento T6. É importante salientar que proporções abaixo de $40 \%$ de lodo de esgoto e de substrato comercial causaram uma diminuição do crescimento da MSPA. Resultados semelhantes foram encontrados por Peroni (2012), que verificou a utilização de substrato comercial e teores abaixo de $40 \%$ de lodo de esgoto na composição do substrato como sendo prejudicial para o desenvolvimento da parte aérea das mudas de Eucalyptus grandis.

Os maiores valores para massa seca de raízes foram encontrados nos tratamentos T5, T6, T7, T9 e T13. Raízes de plantas jovens necessitam do oxigênio para o processo respiratório, que advém do próprio substrato. Com isso, infere-se que há a necessidade de os substratos apresentarem boa aeração para maior crescimento das raízes. Segundo Bunt (1973), nos substratos com adequado espaço de aeração, os valores variam entre 10 e 15\%. Entretanto, percebe-se que, para mudas de S. virgata, valores de espaço de aeração até $25 \%$ e maiores proporções de lodo de esgoto (>40\%) aumentaram a produção de raízes das mudas.

A massa seca total compreende a soma de MSPA e MSR, e quanto maior for esse valor, melhor será a qualidade das mudas produzidas (Cruz, 2006). Para essa característica, as médias variaram de 0,60 g no tratamento T4 a 3,31 g no tratamento T6. Novamente, notou-se a tendência de maiores proporções de lodo de esgoto aumentar o ganho de massa de mudas de S. virgata; além disso, o presente estudo revela que fibra de coco e casca de arroz in natura são resíduos que devem ser evitados para a produção de massa seca das mudas, pois os valores mais baixos foram encontrados com a utilização destes resíduos na formulação dos substrato; provavelmente, isto se deve aos valores inadequados da porosidade total nos substratos formulados com esses resíduos, de acordo com Gonçalves \& Poggiani (1996), e por um baixo teor de nutrientes disponíveis.

A relação altura/diâmetro do coleto (Tabela 7) é reconhecida como um dos melhores indicadores do padrão de qualidade de mudas, sendo, em geral, o mais indicado para determinar a capacidade de sobrevivência no campo (Moreira \& Moreira, 1996), além de usar características não destrutivas. De acordo com Birchler et al. (1998), este índice deve ser menor do que dez para se considerarem mudas com adequado padrão de qualidade. Neste estudo, os valores para esta relação situaram-se entre 3,05 e 5,21 , mostrando que todos os tratamentos estiveram abaixo do limite superior recomendado. Segundo Artur et al. (2007), essa relação reflete o acúmulo de reservas e assegura maior resistência e melhor fixação no solo.

O índice de qualidade dado pela relação massa seca da parte aérea/massa seca de raízes, segundo Caldeira et al. (2008), que avaliaram a produção de mudas de aroeira-vermelha (Schinus terebinthifolius Raddi) em diferentes substratos, deve ser de 2:1. Os autores ainda complementam que é importante analisar essa relação quando as mudas vão para o campo, pois a parte aérea das mudas não deve ser muito superior que a parte aérea da raiz, em função dos possíveis problemas no que se refere à absorção de água para a parte aérea. Considerandose a proporção defendida pelos autores acima, 
Tabela 7. Relação altura/diâmetro do coleto (H/DC), relação massa seca da parte aérea/massa reca da raiz (MSPA/ MSR) e índice de qualidade de Dickson (IQD) de mudas de Sesbania virgata, 150 dias após a semeadura ${ }^{1}$.

Table 7. Height/stem diameter (H/DC), shoot dry mass/root (MSPA/MSR) and Dickson quality index (IQD) of Sesbania virgata seedlings, 150 days after sowing.

\begin{tabular}{|c|c|c|c|}
\hline Tratamento & H/DC & MSPA/MSR & IQD \\
\hline T1 (80\% LE+20\% FC) & $4,50^{\mathrm{b} 1}$ & $1,964^{\mathrm{a}}$ & $0,362^{b}$ \\
\hline $\mathrm{T} 2$ (60\% LE+40\% FC) & $5,21^{\text {a }}$ & $2,066^{a}$ & $0,188^{d}$ \\
\hline T3 (40\% LE+60\% FC) & $4,07^{\mathrm{c}}$ & $1,903^{\text {a }}$ & $0,225^{\mathrm{c}}$ \\
\hline T4 (20\% LE+80\% FC) & $3,43^{d}$ & $1,602^{\mathrm{a}}$ & $0,117^{d}$ \\
\hline T5 (80\% LE+20\% VERM) & $4,56^{\mathrm{b}}$ & $1,443^{a}$ & $0,484^{\mathrm{a}}$ \\
\hline T6 (60\% LE+40\% VERM) & $5,18^{\text {a }}$ & $1,754^{\text {a }}$ & $0,484^{\mathrm{a}}$ \\
\hline T7 (40\% LE+60\% VERM) & $4,65^{\mathrm{b}}$ & $1,338^{a}$ & $0,395^{b}$ \\
\hline T8 (20\% LE+80\% VERM) & $4,62^{b}$ & $1,260^{\mathrm{a}}$ & $0,246^{c}$ \\
\hline T9 (80\% LE+20\% CA in natura) & $4,03^{\mathrm{c}}$ & $1,286^{\mathrm{a}}$ & $0,432^{\text {a }}$ \\
\hline T10 (60\% LE+40\% CA in natura) & $4,07^{\mathrm{c}}$ & $1,245^{b}$ & $0,299^{c}$ \\
\hline T11 (40\% LE+60\% CA in natura) & $4,45^{\mathrm{b}}$ & $0,970^{\mathrm{b}}$ & $0,239^{\mathrm{c}}$ \\
\hline T12 (20\% LE+80\% CA in natura) & $3,05^{\mathrm{d}}$ & $0,787^{b}$ & $0,156^{\mathrm{d}}$ \\
\hline T13 (100\% LE) & $4,78^{\mathrm{b}}$ & $1,314^{\mathrm{a}}$ & $0,350^{\mathrm{b}}$ \\
\hline T14 (100\% SC) & $3,95^{c}$ & $1,464^{\mathrm{a}}$ & $0,261^{\mathrm{c}}$ \\
\hline $\mathrm{F}$ & $* *$ & $* *$ & $* *$ \\
\hline CV\% & 7,61 & 28,01 & 22,53 \\
\hline
\end{tabular}

${ }^{1}$ Médias seguidas de letras iguais, na coluna, não diferem entre si pelo teste de Scott - Knott, em 5\% de significância. ${ }^{* *}$ Significativo $(\mathrm{P}<0,05)$.

os tratamentos que possuem mudas de baixa qualidade são os tratamentos T10, T11 e T12. Contudo, analisando-se que a relação MSPA/MSR depende de duas características destrutivas para sua determinação e que é uma relação contraditória para o crescimento de mudas no campo (Burnett, 1979), não se deve indicá-la para avaliação da qualidade das mudas.

Em relação à característica índice de qualidade de Dickson, as maiores médias encontradas foram nos tratamentos T5, T6 e T9, sendo consideradas mudas com maior equilíbrio de crescimento e, portanto, com qualidade de produção. De acordo com Fonseca (2002), este índice constitui-se num bom indicador, pois pondera características importantes para a avaliação da qualidade das mudas e considera a robustez e o equilíbrio da distribuição da massa na muda.

$\mathrm{Na}$ maioria dos tratamentos analisados, o IQD apresentou média acima de 0,20 , como recomendado por Hunt (1990), mostrando que as mudas produzidas nos diferentes tratamentos apresentam qualidade satisfatória para plantio. Tal resultado está de acordo com trabalhos de Coutinho et al. (2006), que têm apontado efeito benéfico da utilização do lodo de esgoto para compor substratos para mudas de diferentes espécies arbóreas na fase de viveiro. No entanto, mesmo apresentando bom IQD na maioria dos tratamentos, no presente estudo, as mudas não obtiveram grande desempenho nas demais características analisadas, com exceção dos tratamentos T5 e T6, denotando-se a necessidade de conjugar características para uma melhor avaliação de mudas.

Em estudo realizado por Gonçalves et al. (2000), os autores relataram que substratos adequados para a propagação de mudas via semente e estaca podem ser obtidos a partir da mistura de 70 a $80 \%$ de um componente orgânico, com 20 a 30\% de um componente usado para elevar a macroporosidade. Essa tendência observada pelos autores acima referidos concorda com as melhores proporções de substratos verificadas no presente estudo, pois a utilização de 60 a $80 \%$ do lodo de esgoto (componente orgânico) juntamente com $40 \%$ a $20 \%$ de vermiculita, que aumenta a macroporosidade, proporcionou as melhores médias de crescimento da maioria das características morfológicas analisadas.

Resultados semelhantes ao presente estudo foram observados por Nóbrega et al. (2008), com o objetivo de avaliar o efeito do lodo de esgoto misturado em terra de subsolo de Neossolo Quartzarênico 
ou de Latossolo Vermelho Amarelo. Esses autores verificaram que a adição do lodo de esgoto ao solo proporcionou aumento na altura da parte aérea, no diâmetro do coleto, na massa seca de raiz, da parte aérea e total, na razão entre massa seca da parte aérea por massa seca de raiz, no IQD e no número de nódulos, sendo indicada a proporção de 57:43 de lodo de esgoto:solo, recomendada para a produção das mudas de Sesbania virgata.

É possível constatar que vários estudos na literatura mostram que a relação altura/diâmetro do coleto e o IQD são características variáveis (Caldeira et al., 2000, 2008, 2012; Saidelles et al., 2009; Kratz, 2011; Trazzi, 2011; Gomes et al., 2013; Delarmelina et al., 2013). Neste sentido, pode-se inferir que as características em questão podem variar em função da espécie, do manejo das mudas no viveiro, do tipo e da proporção do substrato, do volume do recipiente e, principalmente, de acordo com a idade em que a muda foi avaliada (Caldeira et al., 2012).

\section{CONCLUSÕES}

Os resíduos fibra de coco e casca de arroz in natura não proporcionam crescimento satisfatório das características morfológicas analisadas em mudas de Sesbania virgata.

Os tratamentos com lodo de esgoto e vermiculita em sua composição, principalmente nas proporções de $60 \%$ lodo de esgoto $+40 \%$ de vermiculita, proporcionaram os melhores crescimentos em altura e diâmetro do coleto, e aumentam a produção de massa seca total em mudas de Sesbania virgata.

\section{AGRADECIMENTOS}

À empresa Fibria, pela doação dos materiais utilizados na formulação dos substratos. À Empresa Vale, pela doação das sementes utilizadas no presente estudo.

\section{STATUS DA SUBMISSÃO}

Recebido: 31 jan, 2012

Aceito: 7 abr., 2014

Publicado: 30 jun., 2014

\section{AUTOR(ES) PARA CORRESPONDÊNCIA}

\section{Marcos Vinicius Winckler Caldeira}

Departamento de Ciências Florestais e da Madeira, Centro de Ciências Agrárias, Universidade Federal do Espírito Santo - UFES, CEP 29550-000, Alegre, ES, Brasil e-mail: mvwcaldeira@gmail.com

\section{REFERENNCIAS}

Artur AG, Cruz MCP, Ferreira ME, Barretto VCM, Yagi R. Esterco bovino e calagem para formação de mudas de guanandi. Pesquisa Agropecuária Brasileira 2007; 42(6): 843-850. http://dx.doi. org/10.1590/S0100-204X2007000600011

Birchler T, Rose RW, Royo A, Pardos M. La planta ideal: revision del concepto, parametros definitorios e implementaction practica. Investigacion Agraria, Sistemas y Recursos Forestales 1998; 7(1-2): 109-121.

Brasil. Ministério do Meio Ambiente. Resolução CONAMA n. 375, de 29 de agosto de 2006. Define critérios e procedimentos, para o uso agrícola de lodos de esgoto gerados em estações de tratamento de esgoto sanitário e seus produtos derivados, e dá outras providências. Diário Oficial da União, Brasília, DF (2006 ago. 30). [cited 2013 mar 20]. Available from: http://www.mma.gov.br.

Brasil. Ministério da Agricultura, Pecuária e Abastecimento - MAPA. Instrução normativa SDA n. 17. Métodos analíticos oficiais para análise de substratos para plantas e condicionadores de solo. Diário Oficial da União, Brasília, DF (2007 maio 24).

Bunt AC. Some physical and chemical characteristics of leomless Pat-plant substrates and their relation to plant growth. Plant and Soil 1973; 38: 1954.

Burnett AN. New methods for measuring root growth capacity: their value in assessing lodgepole pine stock quality. Canadian Journal of Forest Research 1979; 9: 6367. http://dx.doi.org/10.1139/x79-011

Caldeira MVW, Peroni L, Gomes DR, Delarmelina WM, Trazzi PA. Diferentes proporções de biossólido na composição de substratos para a produção de mudas de timbó (Ateleia glazioveana Baill). Scientia Forestalis 2012; 40(93): 15-022.

Caldeira MVW, Rosa GN, Fenilli TAB, Harbs RMP. Composto orgânico na produção de mudas de aroeiravermelha. Scientia Agraria 2008; 9(1): 27-33.

Caldeira MVW, Schumacher MV, Barichello LR, Vogel HLM, Oliveira LS. Crescimento de mudas de Eucalyptus saligna Smith em função de diferentes doses de vermicomposto. Revista Floresta 2000; 28(1-2): 19-30.

Carneiro JGA. Produção e controle de qualidade de mudas florestais. Curitiba: UFPR:FUPEF; 1995. 
Chaves LLB, Carneiro JGA, Barroso DG, Leles PSS. Efeitos da inoculação com rizóbio e da adubação nitrogenada na produção de mudas de Sesbania em substrato constituído de resíduos agroindustriais. Revista Árvore 2003; 27(4): 443-449. http://dx.doi. org/10.1590/S0100-67622003000400004

Coutinho MP, Carneiro JGA, Barroso DG, Rodrigues LA, Figueiredo FAMM, Mendonça AVR, et al. Crescimento de mudas de Sesbania virgata (Cav.) Pers. plantadas em uma área degradada por extração de argila. Floresta 2005; 35(2): 231-239.

Coutinho MP, Carneiro JGA, Barroso DG, Rodrigues LA, Siqueira J. Substrato de cavas de extração de argila enriquecido com subprodutos agroindustriais e urbanos para produção de mudas de sesbânia. Revista Árvore 2006; 30(1): 147-153. http://dx.doi.org/10.1590/ S0100-67622006000100018

Cruz CAF. Efeito da adubação nitrogenada na produção de mudas de Sete-Cascas [Samanea inopinata (Harms) Ducke]. Revista Árvore 2006; 30(4): 537-546. http:// dx.doi.org/10.1590/S0100-67622006000400006

Daniel O, Vitorino ACT, Alovisi AA, Mazzochin L, Tokura AM, Pinheiro ER, et al. Aplicação de fósforo em mudas de Acacia mangium Willd. Revista Árvore 1997; 21(2): 163-168.

Delarmelina WM, Caldeira MVW, Faria JCT, Gonçalves EO. Uso de lodo de esgoto e resíduos orgânicos no crescimento de mudas de Sesbania virgata (Cav.) Pers. Revista Agro@mbiente 2013; 7(2): 184-192.

Dickson A, Leaf AL, Hosner JF. Quality appraisal of while spruce and white pine seedling stock in nurseries. Forestry Chronicle 1960; 36: 11-13.

Empresa Brasileira de Pesquisa Agropecuária - EMBRAPA. Manual de análises químicas de solos, plantas e fertilizantes. Brasília; 2009. Embrapa Informação Tecnológica.

Fonseca EP, Valéri SV, Miglioranza E, Fonseca NAN, Couto L. Padrão de qualidade de mudas de Trema micrantha (L.) Blume, produzidas sob diferentes períodos de sombreamento. Revista Árvore 2002; 26(4): 515-523. http://dx.doi.org/10.1590/ S0100-67622002000400015

Gomes DR, Caldeira MVW, Delarmelina WM, Gonçalves EO, Trazzi PA. Lodo de esgoto como substrato para produção de mudas de Tectona grandis L. Cerne 2013; 19(1): 123-131. http://dx.doi.org/10.1590/ S0104-77602013000100015

Gomes JM, Paiva HN. Viveiros florestais: propagação sexuada. Viçosa: UFV; 2006.

Gonçalves JLM, Santarelli EG, Moraes SP No, Manara MP. Produção de mudas de espécies nativas: substrato, nutrição, sombreamento e fertilização. In: Gonçalves JLM, Benedetti V, editors. Nutrição e fertilização florestal. Piracicaba: USP; 2000. p. 309-350.

Gonçalves JLM, Poggiani F. Substrato para produção de mudas florestais. In: Resumos do Congresso Latino Americano de Ciência do Solo; 1996; Águas de Lindóia. Piracicaba: Sociedade Latino Americano de Ciência do Solo; 1996. CD-ROM.
Hunt GA. Effect of styroblock design and cooper treatment on morphology of conifer seedlings. In: Proceedings of Target Seedling Symposium, Meeting of the Western Forest Nursery Associations; 1990; Roseburg. Fort Collins: USDA Forest Service; 1990. p. 218-222. General Technical Report RM-200.

Kratz D. Substratos renováveis na produção de mudas de Eucalyptus benthamii Maiden et Cambage e Mimosa scabrella Benth [dissertação]. Curitiba: Universidade Federal do Paraná; 2011.

Maia AR, Lopes JC, Teixeira CO. Efeito do envelhecimento acelerado na avaliação da qualidade fisiológica de sementes de trigo. Revista Ciência e Agrotecnologia 2007; 31(3): 678-684. http://dx.doi. org/10.1590/S1413-70542007000300012

Melo WJ, Marques MO, Santiago G, Chelli RA. Efeito de doses crescentes de lodo de esgoto sobre frações da matéria orgânica e CTC de um Latossolo cultivado com cana-de-açúcar. Revista Brasileira de Ciência do Solo 1994; 18(3): 449-455.

Morais SMJ, Ataides PRV, Garcia DC, Kurtz FC, Oliveira OS, Watzlawick LF. Uso do lodo de esgoto da Corsan - Santa Maria (RS), comparado com outros substratos orgânicos. Sanare 1996; 6(6): 44-49.

Moreira FMS, Moreira FW. Característica de germinação de 64 espécies de leguminosas florestais nativas da Amazônia, em condições de viveiro. Acta Amazônica 1996; 26(1-2):3-16.

Nóbrega RSA, Paula AM, Boas RCV, Nóbrega JCA, Moreira FMS. Parâmetros morfológicos de mudas de Sesbaniavirgata (Caz.) Persede Anadenantheraperegrina (L.) cultivadas em substrato fertilizado com composto de lixo urbano. Revista Árvore 2008; 32(3): 597-607. http://dx.doi.org/10.1590/S0100-67622008000300020

Peroni L. Substratos renováveis na produção de mudas de Eucalyptus grandis [dissertação]. Alegre: Universidade Federal do Espírito Santo; 2012.

Saidelles FLF, Caldeira MVW, Schirmer WN, Sperandio HV. Casca de arroz carbonizada como substrato para produção de mudas de tamboril-da-mata e garapeira. Semina: Ciências Agrárias 2009; 30(Suppl 1): 1173 $1186 . \quad$ http://dx.doi.org/10.5433/16790359.2009v30n4Sup1p1173

Santos DR, Moreira FMS, Siqueira JO. Fósforo, fungo micorrízico e rizóbio no crescimento, nodulação e fixação biológica do nitrogênio em Sesbania virgata (Cav.) e Sesbania rostrata (Bram). In: Anais do FertBio; 1997; Caxambu.

Trazzi PA. Substratos renováveis na produção de mudas de Tectona grandis Linn F [dissertação]. Jerônimo Monteiro: Universidade Federal do Espírito Santo; 2011. Valeri SV, Corradini L. Fertilização em viveiros para a produção de mudas de Eucalyptus e Pinus. In: Gonçalves JLM, Benedetti V, editors. Nutrição e fertilização florestal. Piracicaba: Instituto de Pesquisas e Estudos Florestais; 2005. p. 167-190. 